\title{
Tumour response to neoadjuvant chemoradiation within lateral pelvic nodes: another step towards precision surgery
}

\author{
R. O. Perez ${ }^{1,2,3} \cdot$ M. D. Daneri ${ }^{1}$ B. Vailati ${ }^{1}$ - G. P. São Julião ${ }^{1}$
}

Received: 24 April 2018 / Accepted: 2 May 2018 / Published online: 10 May 2018

○) Springer International Publishing AG, part of Springer Nature 2018

Lateral lymph-node dissection (LLND) in extraperitoneal rectal cancer has been neglected for a long time in western countries. In addition to being a technically challenging procedure, the high risk of perioperative blood loss and negative effects on urinary and sexual function have contributed to the lack of widespread implementation of LLND in clinical practice outside Japan and Korea [1, 2]. Moreover, with the establishment of multimodal treatment in the management of locally advanced rectal cancer, it has been suggested that patients undergoing preoperative radiation with or without chemotherapy would not require LLND as radiation could potentially sterilize lateral pelvic nodes included in the radiation therapy field [3].

Recently, LLND has attracted significant attention for several reasons. First, the results of a prospective randomized trial in Japan that failed to demonstrate non-inferiority of total mesorectal excision (TME) when compared to TME + LLND in patients with no preoperative evidence of lateral node metastases was quite disturbing [4]. No radiotherapy was used in this trial. The outcomes revealed a higher rate of local recurrence among patients undergoing TME alone. The same trial also demonstrated no differences in male sexual function after LLND when compared to TME alone [5]. Finally, despite the fact that LLND was associated with increased intraoperative bleeding, an elegant case-matched control study in Japan suggested that a minimally invasive approach could potentially solve this particular issue and minimal intraoperative bleeding was reported using the laparoscopic approach [6].

R. O. Perez

rodrigo.operez@gmail.com

1 Angelita and Joaquim Gama Institute, Rua Manoel da Nóbrega 1564, São Paulo, SP 04001-005, Brazil

2 Ludwig Institute for Cancer Research-São Paulo Branch, São Paulo, Brazil

3 Colorectal Surgery Division, University of São Paulo School of Medicine, São Paulo, Brazil
As multimodality treatment of rectal cancer is now mainstreaming in Japan as well, a more selective approach to LLND has been the focus of recent publications. Specific Japanese and Korean centers are suggesting that only in the presence of positive lymph nodes at baseline, should patients undergo "selective" LLND after neoadjuvant chemoradiotherapy (nCRT) [7]. In fact, their own data suggest that nCRT is able to sterilize nearly $50 \%$ of lymph nodes. This means that only $50 \%$ of these patients would still harbor lateral node metastases after nCRT and would ultimately benefit from LLND. One patient out of two would not [7, 8].

In the present edition of Techniques in Coloproctology, Ishihara et al. are taking a step forward in the selective approach for LLND in rectal cancer. With the use of reassessment of tumor response using computed tomography/positron emission tomography (CT/PET) scanning they provide an interesting tool to predict the presence of residual lateral node metastases after nCRT. Using size and metabolic estimate [maximum standardized uptake value $\left.\left(\mathrm{SUV}_{\max }\right)\right]$ cut-offs after nCRT, they were able to predict with high accuracy the presence of lateral node metastasis. This strategy allowed the authors to correctly identify patients that could benefit the most from LLND.

However, prior to implementation of these results in clinical practice, rectal cancer surgeons should consider the following points: First, there seems to be a direct relationship between radiation therapy (RT) dose and tumor response [9]. In an attempt to maximize the chances of sterilizing the lateral lymph nodes, the actual doses of RT that these lymph nodes have received become critical. Moreover, in contemporary multimodality treatment of rectal cancer, there is a place for dose-escalation and boosts given to primary tumors and perhaps also for radiologically positive lateral nodes. The total radiation of 50.4 Gy utilized by Ishihara and colleagues directed to the primary tumor is meaningless with regard to the lateral pelvic nodes. Instead, individual doses to each lateral node compartment become even more relevant. Similarly to what is observed for primary cancers, 
dose-escalation may have been sufficient to sterilize most if not all lateral nodes [10].

Another important consideration is about the lack of information in this article on precise timing of assessment of response and surgery. Again, data about metabolic response from primary cancers have shown that reduction in $\mathrm{SUV}_{\text {max }}$ may be treatment- and time-dependent [11, 12]. Therefore, assessment at different intervals from RT completion may have significantly affected results. Patients scanned immediately after RT completion and undergoing delayed surgery would have been more likely to have false positive size and SUV uptake. In addition, future studies could perhaps investigate the role of dual-point-time imaging, where assessment of SUVs at 1 and $3 \mathrm{~h}$ after fluorodeoxyglucose injection may exhibit distinct patterns to predict response to nCRT [12].

Still, the study by Ishihara et al. opens a new chapter in LLND. It provides definitive insights regarding individualization of rectal cancer management where assessment of tumor response to nCRT becomes central. In the era of precision medicine, which includes organ-preservation among patients with complete clinical response to nCRT, it is very likely that that LLND will be reserved for patients with clear evidence of residual lateral lymph node metastases after nCRT [13]. This will avoid a significant number of unnecessary LLND and restrict this complex procedure to patients that may benefit the most. This is probably a one-way road towards precision surgery.

\section{Compliance with ethical standards}

Conflict of interest The authors declare that they have no conflict of interest.

Ethical standards The present contribtion comply with ethical standards.

Informed consent In the absence of subjects, no informed consent was required.

\section{References}

1. Kusters M, Slater A, Muirhead R et al (2017) What to do with lateral nodal disease in low locally advanced rectal cancer? A call for further reflection and research. Dis Colon Rectum 60:577-585
2. Konishi T, Watanabe T, Nagawa $\mathrm{H}$ et al (2010) Preoperative chemoradiation and extended pelvic lymphadenectomy for rectal cancer: two distinct principles. World J Gastrointest Surg 2:95-100

3. Group MS, Shihab OC, Taylor F et al (2011) Relevance of magnetic resonance imaging-detected pelvic sidewall lymph node involvement in rectal cancer. Br J Surg 98:1798-1804

4. Fujita S, Mizusawa J, Kanemitsu Y et al (2017) Mesorectal excision with or without lateral lymph node dissection for clinical stage II/III lower rectal cancer (JCOG0212): a multicenter, randomized controlled, noninferiority trial. Ann Surg 266:201-207

5. Saito S, Fujita S, Mizusawa J et al (2016) Male sexual dysfunction after rectal cancer surgery: results of a randomized trial comparing mesorectal excision with and without lateral lymph node dissection for patients with lower rectal cancer: Japan Clinical Oncology Group Study JCOG0212. Eur J Surg Oncol 42:1851-1858

6. Yamaguchi T, Konishi T, Kinugasa Y et al (2017) Laparoscopic versus open lateral lymph node dissection for locally advanced low rectal cancer: a subgroup analysis of a large multicenter cohort study in Japan. Dis Colon Rectum 60:954-964

7. Akiyoshi T, Ueno M, Matsueda K et al (2014) Selective lateral pelvic lymph node dissection in patients with advanced low rectal cancer treated with preoperative chemoradiotherapy based on pretreatment imaging. Ann Surg Oncol 21:189-196

8. Kim HJ, Choi GS, Park JS et al (2017) Optimal treatment strategies for clinically suspicious lateral pelvic lymph node metastasis in rectal cancer. Oncotarget 8:100724-100733

9. Appelt AL, Ploen J, Vogelius IR, Bentzen SM, Jakobsen A (2013) Radiation dose-response model for locally advanced rectal cancer after preoperative chemoradiation therapy. Int J Radiat Oncol Biol Phys 85:74-80

10. Habr-Gama A, Sao Juliao GP, Vailati BB et al. (2017) Organ preservation in cT2N0 rectal cancer after neoadjuvant chemoradiation therapy: the impact of radiation therapy dose-escalation and consolidation chemotherapy. Ann Surg. https://doi.org/10.1097/ SLA.0000000000002447

11. Habr-Gama A, Perez RO, Sao Juliao GP et al (2016) Consolidation chemotherapy during neoadjuvant chemoradiation (CRT) for distal rectal cancer leads to sustained decrease in tumor metabolism when compared to standard CRT regimen. Radiat Oncol 11:24

12. Perez RO, Habr-Gama A, Sao Juliao GP et al (2012) Optimal timing for assessment of tumor response to neoadjuvant chemoradiation in patients with rectal cancer: do all patients benefit from waiting longer than 6 weeks? Int J Radiat Oncol Biol Phys 84:1159-1165

13. Sao Juliao GP, Habr-Gama A, Vailati BB, Perez RO (2017) The good, the bad and the ugly: rectal cancers in the twentyfirst century. Tech Coloproctol. https://doi.org/10.1007/s1015 1-017-1651-7 